\title{
Effects of Disorder on the Competition between Antiferromagnetism and Superconductivity
}

\author{
Hiroshi Kohno*, Hidetoshi Fukuyama and Manfred Sigrist ${ }^{1}$ \\ Department of Physics, University of Tokyo, Bunkyo-ku, Tokyo 113, Japan \\ 1 Yukawa Institute, Kyoto University, Kyoto 606-8502, JAPAN
}

(May 30, 2018)

\begin{abstract}
Motivated by the observation of unusual magnetism in $\mathrm{Ce}_{x} \mathrm{Cu}_{2} \mathrm{Si}_{2}(x \sim 1)$, we study the effect of disorder, such as Ce vacancy, on the competition between superconductivity (SC) and antiferromagnetism (AF) on the basis of the phenomenological Ginzburg-Landau theory. Assuming that the AF-SC transition is of first order in clean system, we show that a single impurity in the SC state can induce staggered magnetization by suppressing the SC around it. For finite concentration of impurities, the first-order AF-SC boundary in the clean case is replaced by a finite region where the $\mathrm{SC}$ and the induced AF moments coexist microscopically with spatially varying order parameters. We argue that spin excitation spectrum in the coexistent state has a dual structure of SC gapped mode and the low-energy spin-wave mode. In accordance with the emergence of AF out of SC ground state, the spectral weight will be transferred from the former mode to the latter, keeping the structure of both modes basically unchanged.
\end{abstract}

\section{INTRODUCTION}

The interplay between magnetism and superconductivity (SC) is one of the interesting and profound phenomena ubiquitous in strongly correlated electron systems such as high- $T_{\mathrm{c}}$ cuprates, 'low- $T_{\mathrm{c}}$ ' ruthenates, heavyelectron compounds and molecular solids. Especially, heavy-electron systems often exhibit mysterious magnetism and exotic superconductivity, most of which remain to be characterized [1].

The heavy-electron compound $\mathrm{Ce}_{x} \mathrm{Cu}_{2} \mathrm{Si}_{2}(x \sim 1)$ is located near the phase boundary between superconductivity and antiferromagnetism (AF), and shows an extreme sensitivity to the variation of the Ce concentration $x$ [2 9]. Upon reducing $x$, the ground state is transformed from the spin-singlet SC $(x=1.025,1.00)$ to $\mathrm{AF}$ $(x=0.975)$. For $x=0.99$, several experiments indicate the coexistence of SC and the so-called A-phase, the latter being characterized by the presence of spin fluctuations with unusually low frequency. Recent NQR measurements by Ishida et al. [9] revealed that the energy scale of spin fluctuations in the A-phase is extremely low and of the order of NQR frequency, $\omega_{\mathrm{NQR}} \sim 1-10 \mathrm{MHz}$. Zero-field $\mu \mathrm{SR}$ studies also indicated the existence of random or slowly-fluctuating internal magnetic field [5].

In this letter, we propose that in $\mathrm{Ce}_{x} \mathrm{Cu}_{2} \mathrm{Si}_{2}$ with such a particular type of magnetism an essential role is played by disorder 10. A Ce vacancy, for example, can be the origin of such disorder, which will strongly disturb the electron coherence around it. In the SC state, it will act as a pair breaker and locally reduce the $\mathrm{SC}$ amplitude. It may even induce local magnetization, possibly the staggered one, around it. We present a phenomenological description based on the Ginzburg-Landau (GL) theory for such effects of disorder on the SC state. A key assumption made is that the SC state is in competition with the AF phase with a first-order phase boundary in clean case, which enables the AF phase to nucleate where SC is suppressed. We limit the discussion to absolute zero, namely, the ground state.

Disorder-induced AF in spin-gap systems has been recognized through studies of impurity effects in inorganic spin-Peierls system $\mathrm{CuGeO}_{3}[11,12]$. In this system, a small amount of replacement of $\mathrm{Ge}$ by $\mathrm{Si}$, or $\mathrm{Cu}$ by $\mathrm{Zn}$ induces local staggered moment with local reduction of lattice dimerization, leading to the coexistence of both long-range orderings (LRO's), lattice dimerization and $\mathrm{AF}$, since there is no frustration for $\mathrm{AF}$ as emphasized in ref. [12]. The present work attempts a general treatment for such phenomena, especially in higher dimensions, using the GL theory. Details will appear elsewhere.

\section{CLEAN SYSTEMS}

We begin by considering a clean system, i.e., without impurities, described by the GL free energy density

$$
\begin{aligned}
F= & -\alpha \psi^{2}+\frac{\beta}{2} \psi^{4}-\alpha_{M} M^{2}+\frac{\beta_{M}}{2} M^{4}+\gamma \psi^{2} M^{2} \\
& +\frac{\rho}{2}(\nabla \psi)^{2}+\frac{\rho_{M}}{2}(\nabla M)^{2} .
\end{aligned}
$$

Both order parameters, $\psi$ and $M$, for $\mathrm{SC}$ and $\mathrm{AF}$, respectively, are assumed to be real scalars 13. We are concerned only with their amplitude modulations here and neglect the phase (orientational) degrees of freedom for $\psi(M)$. The constants, $\alpha, \beta, \alpha_{M}, \beta_{M}, \gamma, \rho$ and $\rho_{M}$, are all assumed to be positive. Especially, we consider the case where the ground state is always in a broken-symmetry phase of either SC or AF, which is expressed by the conditions, $\alpha>0$ and $\alpha_{M}>0$. This viewpoint has been taken by Zhang in the $S O(5)$ theory 14 . We also assume that there is no coexistence of $\mathrm{SC}$ and $\mathrm{AF}$ in the clean case, 
which is ensured by the condition, $\gamma^{\prime}>\min \left\{\beta^{\prime}, \beta_{M}^{\prime}\right\}$, where $\gamma^{\prime}=\gamma / \alpha \alpha_{M}, \beta^{\prime}=\beta / \alpha^{2}$ and $\beta_{M}^{\prime}=\beta_{M} / \alpha_{M}^{2}$. Then the ground state is $\mathrm{SC}$ if $\beta^{\prime}<\beta_{M}^{\prime}$ and $\mathrm{AF}$ otherwise.

In the following, we consider the parameter region where the SC state is stabilized in the bulk clean system, but is located near the phase boundary to the AF state. The $\mathrm{SC}$ order parameter takes a constant value $\psi_{0} \equiv \sqrt{\alpha / \beta}$ in the bulk in this case.

\section{SINGLE IMPURITY}

We now introduce a single impurity (at the origin) into the otherwise uniform SC ground state. For $\mathrm{CeCu}_{2} \mathrm{Si}_{2}$, this may represent a Ce vacancy, for example. Assuming that the impurity acts as a local pair breaker for SC and locally favors AF relative to SC, we model its effect by

$$
\begin{aligned}
\alpha & \longrightarrow \alpha(\mathbf{r}) \equiv \alpha\left[1-V_{0} \delta(\mathbf{r})\right], \\
\alpha_{M} & \longrightarrow \alpha_{M}(\mathbf{r}) \equiv \alpha_{M}\left[1+V_{1} \delta(\mathbf{r})\right],
\end{aligned}
$$

where $V_{0}, V_{1} \geq 0$. The GL equations are given by

$$
\begin{aligned}
\frac{\rho}{2} \nabla^{2} \psi(\mathbf{r}) & =\left[-\alpha(\mathbf{r})+\gamma M^{2}\right] \psi+\beta \psi^{3}, \\
\frac{\rho_{M}}{2} \nabla^{2} M(\mathbf{r}) & =\left[-\alpha_{M}(\mathbf{r})+\gamma \psi^{2}\right] M+\beta_{M} M^{3} .
\end{aligned}
$$

Equation (4) describes the suppression of the SC order parameter $\psi$ around the impurity. If we neglect the $\gamma$ term for simplicity [15], the solution is written as

$$
\psi(\mathbf{r})=\sqrt{\alpha / \beta} f\left(r ; \xi, V_{0}\right)
$$

with $\xi=\sqrt{\rho / 2 \alpha}$ and $r=|\mathbf{r}|$. Qualitative features of the function $f$ are as follows. It is suppressed appreciably from the bulk value of 1 only within a region $r \lesssim \xi$, where it decreases almost linearly with $r$ as $r \rightarrow 0$. The value $f(r=0)$ at the origin is a monotonically decreasing function of $V_{0}$; it is 1 for $V_{0}=0$ and vanishes as $V_{0} \rightarrow \infty$.

Equation (5) describes the possible emergence of staggered moment $M$ around the impurity. To study this, we first drop the non-linear term $\left(\sim M^{3}\right)$ and consider the following eigenvalue problem

$$
\left[-\frac{\rho_{M}}{2} \nabla^{2}+U_{M}(\mathbf{r})\right] M(\mathbf{r})=\varepsilon M(\mathbf{r})
$$

where the 'potential' $U_{M}(\mathbf{r})$ is given by

$$
U_{M}(\mathbf{r})=\tilde{\alpha}_{M}-\gamma\left[\psi_{0}^{2}-\psi^{2}(\mathbf{r})\right]-\alpha_{M} V_{1} \delta(\mathbf{r}) .
$$

In eq.(8), the first term, $\tilde{\alpha}_{M} \equiv-\alpha_{M}+\gamma \psi_{0}^{2}$, is positive [16], which assures the local stability ('local' in the $\psi-M$ space) of the pure SC state in the clean case. The second and the third terms represent 'attractive potential' due to the suppression of $\psi$, and the direct coupling to the impurity, respectively. If we have a non-zero solution
$M(\mathbf{r})$ with negative 'energy' $\varepsilon$, this signals the appearance or 'nucleation' of the AF phase around the impurity in the otherwise SC system 17]. Its spatial pattern is given by the corresponding eigenfunction (with lowest eigenvalue, $\varepsilon$ ) and the amplitude is determined by balancing the quadratic term $\left(\sim \varepsilon M^{2}\right)$ with the quartic term $\left(\sim \beta_{M} M^{4}\right)$, which leads to $M \sim \sqrt{-\varepsilon / \beta_{M}}$.

As we have seen, the characteristic length-scale for $\psi$ is given by $\xi$. On the other hand, spatial variation of $M$ occurs on the scale, $\xi_{M}=\sqrt{\rho_{M} / 2 \alpha_{M}}$. 118. Depending on their ratio, $\xi / \xi_{M}$, there are two different regimes, namely, $\xi \ll \xi_{M}$ (type I) and $\xi \gg \xi_{M}$ (type II). The intermediate regime $\left(\xi \sim \xi_{M}\right)$ may be the case where the $S O(5)$ symmetry, a rotational symmetry in the $\psi-M$ space, is expected. We examine these three cases in the following.

(i) Type I $\left(\xi \ll \xi_{M}\right)$ : The suppression of $\psi$ is limited to a small region compared to $\xi_{M}$. Its effect on $M$ can be shown to be negligible, and the $V_{1}$-term is necessary for $M$ to nucleate. Rewriting eq.(7) as

$$
\left(-\nabla^{2}+\kappa_{M}^{2}\right) M(\mathbf{r})=v_{1} M_{0} \delta(\mathbf{r}),
$$

with $v_{1}=V_{1} / \xi_{M}^{2}, \kappa_{M}=\kappa_{M}(\varepsilon) \equiv \sqrt{2\left(\tilde{\alpha}_{M}-\varepsilon\right) / \rho_{M}}$, and $M_{0} \equiv M(\mathbf{r}=\mathbf{0})$, the solution is obtained as

$$
M(\mathbf{r})=v_{1} M_{0} G\left(\mathbf{r} ; \kappa_{M}\right),
$$

where $G(\mathbf{r} ; \kappa)$ is the Green's function satisfying $\left(-\nabla^{2}+\right.$ $\left.\kappa^{2}\right) G(\mathbf{r} ; \kappa)=\delta(\mathbf{r})$. The explicit form is given by

$$
G(\mathbf{r} ; \kappa)=\mathrm{e}^{-\kappa r} / 2 \kappa, \quad K_{0}(\kappa r) / 2 \pi, \quad \mathrm{e}^{-\kappa r} / 4 \pi r,
$$

for dimensions $d=1,2$ and 3 , respectively, where $K_{0}$ is the modified Bessel function of rank zero. (The present Ce-compound corresponds to $d=3$.) Equation (10) expresses a nucleated staggered magnetization if the solution exists such that $M_{0} \neq 0$ and $\varepsilon<0$.

The condition for $M_{0} \neq 0$ is obtained from (10) by putting $\mathbf{r}=\mathbf{0}$ and dividing both sides by $M_{0}$ as

$$
1=v_{1} G\left(r=a ; \kappa_{M}(\varepsilon)\right) .
$$

This is an eigenvalue equation for $\varepsilon$. For $d=2$ and 3 , the right hand side is evaluated by introducing a cut-off $a$ which will be of the order of the lattice constant [19]. The nucleation of $M$ occurs for $v_{1}>v_{1, \text { cr }}$ where

$$
v_{1, \mathrm{cr}}=2 / \tilde{\xi}_{M}, \quad 2 \pi / K_{0}\left(a / \tilde{\xi}_{M}\right), \quad 4 \pi a \exp \left(a / \tilde{\xi}_{M}\right),
$$

for $d=1,2$ and 3, respectively, with $\tilde{\xi}_{M}=\sqrt{\rho_{M} / 2 \tilde{\alpha}_{M}}$.

(ii) Type II $\left(\xi \gg \xi_{M}\right)$ : In this case, $\psi$ is suppressed in a wider region compared to $\xi_{M}$, and it is possible for $M$ to nucleate even without the $V_{1}$-term. As an example we consider the case $V_{0} \rightarrow \infty$ and $V_{1}=0$. Then the potential for $M$ is given by a harmonic one generated by the linear $r$-dependence of $\psi(\mathbf{r})$ around the impurity. If we assume such harmonic potential in the entire space, the condition for the nucleation of $M$ is obtained as $\xi_{M}<\xi / d^{2}$. 
(iii) ' $S O(5)$ symmetric' case $\left(\xi=\xi_{M}\right)$ : In case both length-scales are comparable, we must solve the coupled equations for $\psi$ and $M$ simultaneously. However, a simplification is possible if the 'anisotropy' is weak in the $\psi-M$ space. We define the case what may be called ' $S O(5)$ symmetric' by $\xi=\xi_{M}$. We then introduce the angular $(\theta)$ and radial $(R)$ variables by $\left(\alpha^{1 / 2} \psi, \alpha_{M}^{1 / 2} M\right)=(R \cos \theta, R \sin \theta)$, to obtain

$$
F=\xi^{2}\left[(\nabla R)^{2}+R^{2}(\nabla \theta)^{2}\right]-R^{2}+\frac{1}{2} \beta(\theta) R^{4}
$$

for clean systems, where $\beta(\theta)=\beta_{0}+g \cos 2 \theta+B \sin ^{2} 2 \theta$, $\beta_{0}=\frac{1}{2}\left(\beta^{\prime}+\beta_{M}^{\prime}\right), g=\frac{1}{2}\left(\beta^{\prime}-\beta_{M}^{\prime}\right)$, and $B=\frac{1}{2}\left(\gamma^{\prime}-\beta_{0}\right)$. The terms with $g$ and $B$ represent, respectively, the 'anisotropy energy' and the energy barrier between SC and AF. $(g<0$ favors SC relative to AF.) The condition of weak anisotropy, $|g|,|B| \ll \beta_{0}$, restricts the low-energy configurations to the manifold $R=R(\theta) \equiv$ $\beta(\theta)^{-1 / 2}$, which as a function of $\theta$ has small variation compared to the average $\sim R_{0} \equiv \beta_{0}^{-1 / 2}$. Introducing the impurity potential by $F_{\mathrm{imp}}=V R^{2} \cos 2 \theta \delta(\mathbf{r})$, and considering the spatial variation of the order parameters, we again recognize two length-scales, $\xi_{R} \sim \xi$ and $\xi_{\theta} \sim \xi \sqrt{\beta_{0} / \max \{|g|,|B|\}}$, now associated with variables $R$ and $\theta$, respectively. From the above condition, we have $\xi_{R} \ll \xi_{\theta}$. Therefore, while both $R$ and $\theta$ deviates from the bulk values in the vicinity of the impurity $\left(r<\xi_{R}\right)$, for $r>\xi_{R}$, only $\theta$ governs the spatial variation and $R$ follows $\theta$ completely as $R=R(\theta)$ given above. The behaviours in the region $r>\xi_{R}$ will hence be described by the effective free energy, $F_{\theta}$ :

$$
F_{\theta} / R_{0}^{2}=\xi^{2}(\nabla \theta)^{2}+g(\mathbf{r}) \cos 2 \theta+B^{\prime} \sin ^{2} 2 \theta,
$$

where $g(\mathbf{r})=\left(g / 2 \beta_{0}\right)+V \delta(\mathbf{r}), B^{\prime}=B / 2 \beta_{0}$. For $B=0$ and $d=1$, the present problem is equivalent to that of the disordered spin-Peierls system 12,20]. The one with $B=0$ and $d=2$ (with $V=0$ ) corresponds to the $S O(5)$ description proposed for high- $T_{\mathrm{c}}$ cuprates [14].

\section{FINITE CONCENTRATION OF IMPURITIES}

In the presence of many impurities (located at $\left\{\mathbf{R}_{i}\right\}$ ), the solution is written as

$$
\mathbf{M}(\mathbf{r})=\sum_{i} \mathbf{m}_{i} M_{1}\left(\mathbf{r}-\mathbf{R}_{i}\right)
$$

in the dilute concentration limit. Here $M_{1}$ is the solution for the single-impurity problem obtained in the previous section, and we have recovered the vector nature of the $\mathrm{AF}$ order parameter. The unit vector, $\mathbf{m}_{i}$, represents the direction of the staggered moment nucleated around the impurity at $\mathbf{R}_{i}$, and the interaction among them will be described by

$$
F_{\mathrm{m}}=-\sum_{i<j} J_{i j} \mathbf{m}_{i} \cdot \mathbf{m}_{j}
$$

The effective exchange constants

$$
J_{i j}=(-2 \varepsilon) \int \mathrm{d} \mathbf{r} M_{1}\left(\mathbf{r}-\mathbf{R}_{i}\right) M_{1}\left(\mathbf{r}-\mathbf{R}_{j}\right)+\ldots
$$

do not have frustration in the present model focussing on the commensurate AF, but will allow it in general. For depleted quantum spin systems, similar models have been obtained previously [21].

Orientational fluctuations of $\mathbf{m}_{i}$ 's will produce lowenergy spin excitations even when the LRO of SC is not completely destroyed by the emergence of $M$. If $\mathbf{m}_{i}$ 's align and exhibit AFLRO, their fluctuations will show up as a gapless Goldstone (spin-wave) mode. We note that the coexistence of both types of LRO, SC and impurityinduced $\mathrm{AF}$, is possible due to the spatial variation of the two order parameters in spite of their competition. This general aspect has been first noticed in studies on disordered spin-Peierls systems. 12] If AFLRO failed to be established for some reasons (see below), fluctuations of $\mathbf{m}_{i}$ 's will remain as a diffusive mode with very low energy with the total spectral weight being proportional to $M_{1}^{2}$ and the concentration of impurities.

\section{DISCUSSION}

We have given an analysis based on the GL theory of the effect of disorder in the SC ground state which is in competition with the AF state. Our interest is focussed on the nucleation of the AF phase around disorder centers. As the concentration of impurities is increased, the original SC state will first change into the microscopically coexistent state with nucleated staggered moments, and finally be transformed into the complete AF. The firstorder AF-SC boundary in the clean case thus turns into a finite region of coexistence.

Analogously to disordered spin-Peierls systems [22,23], the present coexistent state has spin excitations with two distinct features, the SC gapped excitation due to quasiparticles and the gapless spin-wave mode, well separated from each other by a 'transparent' region (see Fig.11). In accordance with the emergence of AF out of SC ground state, the spin excitation spectrum will change mainly through the transfer of the spectral weight from the gapped mode to the spin-wave mode, keeping the structure and energy scales of both modes essentially unchanged, with a possible reduction of the SC gap and an increase of the spin-wave velocity. 

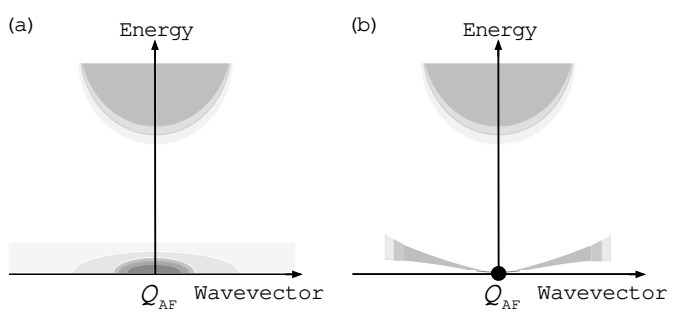

FIG. 1. Spin excitation spectrum in the coexistent phase of SC and disorder-induced staggered magnetization. It has a dual structure of SC gapped mode from quasiparticles and the low-energy mode from nucleated staggered moments. (a) In the absence of AFLRO, low-energy excitations are diffusive. (b) In the presence of AFLRO, low-energy excitations are Goldstone (spin wave) modes. The dot at $Q_{\mathrm{AF}}$ indicates the Bragg spot of AF. The energy scale for the low-energy mode is exaggerated.

We would like to propose that the above picture describes well the evolution of the ground state from SC to $\mathrm{AF}$ in $\mathrm{Ce}_{x} \mathrm{Cu}_{2} \mathrm{Si}_{2}$. Especially, we propose that the Aphase realized near the phase boundary is characterized by a coexistence phase of SC and the nucleated staggered moments. Experiments so far performed on samples in the A-phase did not detect any signals of magnetic LRO [24], but revealed the existence of dynamical fluctuations with very low frequency of NQR scale [9]. Such lowenergy fluctuations will be naturally understood as orientational fluctuations of the nucleated staggered moments around impurities. In the absence of true AF LRO, they behave as diffusive (Fig.1(a)). The spectral weight can be substantial at low energy since they are transformed into a Bragg spot and collective spin-wave modes as AF LRO is reached (Fig.1(b)).

The nucleation of staggered moments will occur at some finite temperature. Then what is their fate in the low-temperature limit where thermal fluctuations are diminished? There are several possibilities depending on the interaction $J_{i j}$ among them; (i) Constructive interaction (without frustration) leads to AFLRO, (ii) the presence of frustration leads to a spin-glass state, (iii) even if there is no frustration, the interaction among them is so weak and their orientations freeze almost randomly and independently, resulting in a spin-glass-like state, and (iv) they continue fluctuating due to quantum effects. The spin-Peierls system $\mathrm{Cu}_{1-x} \mathrm{Zn}_{x} \mathrm{GeO}_{3}$ offers an example of case (i), where AF LRO has been observed in samples with $x$ being as low as $1.12 \times 10^{-3}$ with Néel temperature $T_{N}=0.0285 \mathrm{~K}$. 25] For $\mathrm{Ce}_{x} \mathrm{Cu}_{2} \mathrm{Si}_{2}$ with $x=0.99$, the NQR experiment has been done down to temperature $T=0.012 \mathrm{~K}$, which suggests the case (ii) or (iii). If the nucleated staggered moments are incommensurate [26], their interaction will be frustrated, leading to the case (ii).

Our present picture predicts the spectral weight transfer between the 'rigid bands' of gapped mode and the 'spin-wave' mode (Goldstone or diffusive, depending on the presence or absence of AF LRO) as the ground state is tuned from $\mathrm{SC}$ to $\mathrm{AF}$ through the coexistent phase. It will be interesting to explore experimentally this spectralweight transfer by sweeping through the phase transition via, for example, pressure as proposed in ref. [9].

In the coexistent state proposed in this paper, $\mathrm{SC}$ and $\mathrm{AF}$ are in competition even though they 'coexist'. The $\mu \mathrm{SR}$ studies [6.7] seem to have revealed their competing nature experimentally, but then concluded a spatial seggregation of the two phases. Before deriving such a conclusion, an analysis from the present viewpoint may be necessary.

In conclusion, we have examined a possible nucleation of the $\mathrm{AF}$ phase due to disorder in the otherwise pure SC state and proposed that such a coexistent phase is responsible for the peculiar magnetism in the so-called A-phase of $\mathrm{Ce}_{x} \mathrm{Cu}_{2} \mathrm{Si}_{2}$. Such effects of disorder on the interplay between magnetic ordering and superconductivity (or spin gap in general) will be quite ubiquitous in strongly correlated systems and deserve attention in interpreting experimental data.

We would like to thank Professor Y. Kitaoka for a nice introduction to $\mathrm{Ce}_{x} \mathrm{Cu}_{2} \mathrm{Si}_{2}$, as well as for valuable comments.

[1] For a review on heavy-electron superconductivity, N. Grewe and F. Steglich, in Handbook on the Physics and Chemistry of Rare Earths, eds. K.A. Gschneidner, Jr., and L. Eyring (North Holland, Amsterdam), Vol. 14, p.343; F. Steglich, U. Ahlheim, C.D. Bredl, C. Geibel, M. Lang, A. Loidl and G. Sparn, in Frontiers in Solid State Science, eds. L.C. Gupta and M.S. Multani (World Scientific, Singapore, 1992), p.527; R.H. Heffner and M.R. Norman, Comments Condens. Matter Phys. 17 (1996) 361.

[2] F. Steglich, J. Aarts, C.D. Bredl, W. Lieke, D. Meschede, W. Franz and H. Schf̈er, Phys. Rev. Lett. 43 (1979) 1892.

[3] Y. Kitaoka, H. Nakamura, T. Iwai, K. Asayama, U. Ahlheim, C. Geibel, C. Schank and F. Steglich, J. Phys. Soc. Jpn. 60 (1991) 2122.

[4] H. Nakamura, Y. Kitaoka, T. Iwai, H. Yamada and K. Asayama, J. Phys.: Condens. Matter 4 (1992) 473.

[5] Y.J. Uemura, W.J. Kossler, X.H. Yu, H.E. Schone, J.R. Kempton, C.E. Stronach, S. Barth, F.N. Gygax, B. Hitti, A. Schenck, C. Baines, W.F. Lankford, Y. Ōnuki and T. Komatsubara, Phys. Rev. B39 (1989) 4726.

[6] G.M. Luke, A. Keren, K. Kojima, L.P. Le, B.J. Sternlieb, W.D. Wu, Y.J. Uemura, Y. Ōnuki and T. Komatsubara, Phys. Rev. Lett. 73 (1994) 1853.

[7] R. Feyerherm, A. Amato, C. Geibel, F.N. Gygax, P. Hellmann, R.H. Heffner, D.E. MacLaughlin, R. MüllerReisener, G.J. Nieuwenhuys, A. Schenck and F. Steglich, 
Phys. Rev. B56 (1997) 699.

[8] For a review on $\mu$ SR study, see A. Amato, Rev. Mod. Phys. 69 (1997) 1119.

[9] K. Ishida, Y. Kawasaki, K. Tabuchi, K. Kashima, Y. Kitaoka, K. Asayama, C. Geibel and F. Steglich, preprint.

[10] H. Fukuyama, A paper reported at 7th ISSP International Symposium "Frontiers in Neutron Scattering Research", to appear in J. Phys. Chem. Solids (1999) condmat/9812294).

[11] L.P. Regnault, J.P. Renard, G. Dhalenne and A. Revcolevschi, Europhys. Lett. 32 (1995) 579; M. Hase, K. Uchinokura, R.J. Birgeneau, K. Hirota and G. Shirane, J. Phys. Soc. Jpn. 65 (1996) 1392.

[12] H. Fukuyama, T. Tanimoto and M. Saito, J. Phys. Soc. Jpn. 65 (1996) 1182.

[13] We assume spin-singlet pairing (with one-dimensional representation) for $\mathrm{SC}$, and commensurate structure for AF.

[14] S.C. Zhang, Science 275 (1997) 1089.

[15] The effect of the $\gamma$ term on $\psi$ can be taken into account as follows. For the type I case (defined below), we substitute $\alpha-\gamma M^{2}(\mathbf{r})$ for $\alpha$ in the sense of an 'adiabatic' approximation. For the type II case, it may be modeled by the $\delta$-function potential for $\psi$, which just renormalizes $V_{0}$.

[16] This follows from the assumptions mentioned in $\S 2$.

[17] The appearance of a bound state is not a sufficient condition since there is a positive offset $\tilde{\alpha}_{M}$ to the 'energy' $\varepsilon$. A bound state with positive 'energy' $\varepsilon$ is an excited state.

[18] Actually, however, $\xi$ and $\xi_{M}$ depend on the state, or the values of the order parameters. For example, in a clean SC state where $\psi \equiv \psi_{0}$ and $M \equiv 0$, they are given by $\sqrt{\rho / 4 \alpha}$ and $\tilde{\xi}_{M}=\sqrt{\rho_{M} / 2 \tilde{\alpha}_{M}}$, respectively. In case where the order parameters have large spatial variation (which is our present concern), $\xi$ and $\xi_{M}$ should be obtained after solving the GL equations. In fact, the length scale for $M$ is given by $\kappa_{M}^{-1}(\varepsilon)$ defined below. However, the following arguments remain valid.

[19] One way to see this is to consider the fourth-order term in gradient, $F \sim \rho_{M}(\nabla M)^{2}+\rho_{M 4}\left(\nabla^{2} M\right)^{2}$; then we have $a \sim \sqrt{\rho_{M 4} / \rho_{M}}$.

[20] M. Saito, J. Phys. Soc. Jpn. 67 (1998) 2477.

[21] M. Sigrist and A. Furusaki, J. Phys. Soc. Jpn. 65 (1996) 2385; N. Nagaosa, A. Furusaki, M. Sigrist and H. Fukuyama, ibid 3724.

[22] M.C. Martin, M. Hase, K. Hirota, G. Shirane, Y. Sasago, N. Koide and K. Uchinokura, Phys. Rev. B56 (1997) 3173; K. Hirota, M. Hase, J. Akimitsu, T. Matsuda, K. Uchinokura and G. Shirane, J. Phys. Soc. Jpn. 67 (1998) 645.

[23] M. Saito and H. Fukuyama, J. Phys. Soc. Jpn. 66 (1997) 3259 .

[24] J. Flouquet et al., private communications.

[25] K. Manabe, H. Ishimoto, N. Koide, Y. Sasago and K. Uchinokura, Phys. Rev. B58 (1998) R575.

[26] The incommensurate nature of the magnetic ordering in $\mathrm{Ce}_{0.975} \mathrm{Cu}_{2} \mathrm{Si}_{2}$ has been indicated experimentally in ref [9]. 